\title{
U tilidad de la tomografía computarizada de emisión de fotón único sincronizada con el electrocardiograma para la detección de isquemia miocárdica silente en diabéticos tipo 2
}

\author{
Yamilé Peña $\mathrm{Q}^{1 \mathrm{a}}$, Marco Antonio Coca $\mathrm{P}^{1}$, \\ Juan Felipe Batista $C^{1}$, José Fernández-Britto $R^{2}$, \\ Rodobaldo Q uesada P1, Andria Peña $C^{1}$. \\ Gated single photon emission \\ computer tomography for the detection \\ of silent myocardial ischemia
}

Background: Asymptomatic patients with severe coronary atherosclerosis may have a normal resting electrocardiogram and stress test. Aim: To assess the yield of Gated Single Photon Emission Computer Tomography (SPECT) for the screening of silent myocardial ischemia in type 2 diabetic patients. Material and methods: Electrocardiogram, stress test and gated-SPECT were performed on 102 type 2 diabetic patients aged $60 \pm 8$ years without cardiovascular symptoms. All subjects were also subjected to a coronary angiography, whose results were used as gold standard. Results: Gated-SPECT showed myocardial ischemia on $26.5 \%$ of studied patients. The sensibility, specifity, accuracy, positive predictive value and negative predictive value were $92.3 \%, 96 \%, 95 \%, 88.8 \%, 97.3 \%$, respectively. In four and six patients ischemia was detected on resting electrocardiogram and stress test, respectively. Eighty percent of patients with doubtful resting electrocardiogram results and $70 \%$ with a doubtful stress test had a silent myocardial ischemia detected by gated-SPECT. There was a good agreement between the results of gated-SPECT and coronary angiography ( $\mathrm{k}=0.873)$. Conclusions: Gated-SPECT was an useful tool for the screening of silent myocardial ischemia (Rev Méd Chile 2009; 137: 1023-30).

(Key words: Diabetes mellitus, type 2; Myocardial ischemia; Tomography, emission-computed, single-photon)

Recibido el 13 de noviembre, 2008. Aceptado el 22 de junio, 2009.

${ }^{1}$ Centro de Investigaciones Clínicas, Ciudad de La Habana, Cuba. ${ }^{2}$ Centro de Investigación y Referencia de Aterosclerosis, Ciudad de La Habana, Cuba.

${ }^{a}$ Master en Investigación en Aterosclerosis. 
$\mathrm{L}$ a isquemia miocárdica silente es aquella documentada de forma objetiva mediante métodos diagnósticos en ausencia de angina o equivalentes anginosos. Su prevalencia es difícil de estimar en la población general, pero aumenta con la presencia de factores de riesgo coronario como la hipertensión arterial y la diabetes mellitus ${ }^{1}$.

Los pacientes diabéticos poseen un riesgo de morir de enfermedad coronaria $2-4$ veces mayor que las personas sin diabetes ${ }^{2}$. Estudios anteriores han demostrado que en el paciente diabético los síntomas no constituyen una guía adecuada para el diagnóstico y seguimiento de las enfermedades cardiovasculares $^{3-5}$, es por ello que la detección de cardiopatía isquémica mediante diferentes métodos no invasivos debe formar parte de un cuidado rutinario en estos pacientes.

Los estudios de perfusión miocárdica tienen un papel importante en el diagnóstico de la isquemia que aún no es evidenciada clínicamente y que no posee manifestaciones en otros exámenes como el electrocardiograma y la prueba de esfuerzo. La tomografía computarizada por emisión de fotón único sincronizada con el electrocardiograma (gated-SPECT) es una técnica no invasiva en la que no sólo se valora la perfusión miocárdica, sino que además se obtienen datos de la función ventricular ${ }^{6,7}$, posee una alta capacidad diagnóstica en pacientes con manifestaciones clínicas ${ }^{8-10}$. El objetivo del presente trabajo fue determinar la utilidad de la tomografía computarizada de emisión de fotón único sincronizada al electrocardiograma para la detección de isquemia miocárdica silente en pacientes diabéticos tipo 2 sin clínica cardiovascular.

\section{MATERIAL Y MÉTODO}

Se seleccionaron 102 pacientes de seis áreas de salud de Ciudad de La Habana.

\section{Criterios de inclusión:}

Diabéticos tipo 2 de ambos sexos, mayores de 18 años, sin clínica cardiovascular (ausencia de síntomas y signos de cardiopatía isquémica) que previamente no poseyeran diagnóstico de enfermedad cardiaca isquémica. Participación voluntaria en el ensayo expresada por escrito mediante un consentimiento informado.
Criterios de exclusión:

Embarazo o lactancia. Limitaciones y enfermedades severas que impidan la realización de ejercicios en estera rodante. Electrocardiograma basal con bloqueo completo de rama izquierda.

Los pacientes reunieron los criterios recomendados por la Asociación Americana de Diabetes ${ }^{11}$ para screening de enfermedad cardiovascular. El $92,2 \%$ llevaba tratamiento con dieta o hipoglicemiantes orales, 51,9\% tenía un tiempo de evolución de la diabetes superior a los 10 años.

Factores de riesgo. Fumadores 33\%, hipertensión arterial $76,4 \%$, sedentarismo $66 \%$, obesidad 34\%, enfermedad vascular periférica $18,9 \%$, antecedentes patológicos familiares de cardiopatía isquémica $38,7 \%$, retinopatía $2,8 \%$, nefropatía $1,8 \%$.

Se realizó electrocardiograma, prueba de esfuerzo y tomografía computarizada de emisión de fotón único sincronizada al electrocardiograma (gated-SPECT). La coronariografía constituyó el criterio de confirmación.

\section{Medios diagnósticos}

Electrocardiograma basal (EKG). Se consideró Positivo: inversión simétrica de la onda $\mathrm{T}$, infradesnivel del segmento ST $\geq 1 \mathrm{~mm}$, presencia de onda $\mathrm{Q}$ patológica en dos o más derivaciones eléctricas. Dudoso: Presencia de alteraciones inespecíficas del electrocardiograma (hipertrofia ventricular izquierda, eje a la izquierda, trastornos de repolarización y arritmias). Negativo: Ausencia de signos electrocardiográficos de cardiopatía isquémica.

Prueba de esfuerzo electrocardiográfica. Se empleó el protocolo de Bruce modificado. Se consideró Positiva: Criterio clínico: aparición de angina de esfuerzo. Criterio electrocardiográfico: Infradesnivel horizontal o descendente del segmentoST $\geq 0,1 \mathrm{mV}$ a los $0,8 \mathrm{~s}$ del punto $\mathrm{J}$, durante el ejercicio. Dudosa: Alteraciones del electrocardiograma (depresión del punto j con ST ascendente en ausencia de angina, ausencias de cambios eléctricos sin que alcanzara una adecuada frecuencia cardiaca, aparición de arritmia durante el ejercicio. Negativa: Ausencia de cambios patológicos del ST durante el ejercicio que alcanzó frecuencia cardiaca submáxima (220 - edad x 85). 
Gated-SPECT. Se empleó el protocolo de días diferentes variante estrés-reposo. El ejercicio fue realizado en una estera rodante empleando el protocolo de Bruce modificado. Se realizaron tomografías sincronizadas al monitor de electrocardiograma de 32 proyecciones de $50 \mathrm{~s}$ cada una, en órbita circular de 180 grados, con matriz de $64 \times 64$ pixeles una hora posterior a la inyección endovenosa de $1110 \mathrm{MBq}$ (30 mCi) de ${ }^{99 \mathrm{~m}} \mathrm{Tc}-\mathrm{MIBI}$ por cada paciente. Las imágenes fueron procesadas en una estación de trabajo PowerVision (SMV, Canadá) empleando el software Emory Cardiac Tool Box (Universidad de Emory, EE.UU.).

Evaluación de la perfusión. Se realizó examen visual y cuantitativo de los cortes de eje largo vertical, eje largo horizontal y eje corto perpendicular, así como de 17 segmentos del ventrículo izquierdo en la imagen de mapa polar según recomendaciones de las guías de American Society of Nuclear Cardiology (ASNC) y American College of Cardiology (ACC) ${ }^{12}$. Los defectos de perfusión se valoraron teniendo en cuenta la extensión, intensidad, reversibilidad y afectación de uno o más territorios. Se clasificaron en no extensos (un solo segmento involucrado) y en extensos (dos o más segmentos involucrados), la intensidad se informó de acuerdo al por ciento de captación en comparación con el área de mayor captación en la imagen polar del ventrículo izquierdo (defecto ligero captación entre 60\% y $69 \%$, moderado entre $50 \%$ y $59 \%$ y severo inferior a $50 \%{ }^{12}$. La reversibilidad se catalogó como total, parcial o nula.

Se consideró gated-SPECT positivo: Defecto de captación segmentario o regional que abarcara al menos dos sectores por debajo de $70 \%$ del máximo nivel de captación en la imagen polar del ventrículo izquierdo. Los totalmente reversibles se consideraron patrones isquémicos, los parcialmente reversibles se interpretaron como áreas en las que coexiste tejido cicatrizal o como isquemias intensas. Los defectos con captaciones inferior a $30 \%$ o entre $30 \%$ y $49 \%$ que no se modificaban con el reposo fueron considerados escaras (necróticos).

Gated-SPECT negativo: Ausencia de defectos por debajo de $70 \%$. Los defectos ligeros que no se modificaban con el reposo en región inferior y anterior fueron interpretados como atenuaciones.
Evaluación de la función. Se calculó la fracción de eyección del ventrículo izquierdo global, se consideró normal los valores por encima de 50\%, se calcularon los volúmenes telediastólicos y telesistólicos según Faber TL et al ${ }^{13}$. Se valoró el engrosamiento sistólico de las paredes ventriculares según modo cine (visual) y mapa de engrosamiento parietal (cuantitativo), el movimiento regional de paredes, la evaluación visual del volumen, la evaluación visual del histograma de fase de contracción ventricular y la evaluación de los índices de función diastólica. También fueron valorados otros parámetros funcionales si estaban presentes, tales como: detección de aquinesias, hipoquinesias y disquinesias del VI, modificación de los diámetros cavitarios en telesístole y telediástole.

Coronariografía. Se empleó método angiográfico cuantitativo (vía femoral), se consideró lesiones significativas las obstrucciones $\geq 50 \%$.

Análisis estadístico. Se calcularon estadísticas descriptivas (media y desviación estándar) para todas las variables continuas. Se determinó el número y porcentaje de pacientes con resultados positivos, dudosos y negativos de isquemia miocárdica silente en cada técnica diagnóstica. Se construyeron tablas de doble entrada que relacionan las variables binarias con la condición de gated-SPECT positivo y gated-SPECT negativo, calculando en cada caso el coeficiente de contingencia (CC) para comprobar la existencia de asociación y la significación $(\mathrm{p}<0,05)$.

Se calcularon los valores de sensibilidad, especificidad, exactitud, valor predictivo positivo (VPP) y valor predictivo negativo (VPN) del gatedSPECT. Fue determinado el coeficiente kappa ( $\kappa)$ para establecer la concordancia entre los resultados del gated-SPECT y la coronariografía. Para ello se empleó el programa SPSS v15.0.

\section{Resultados}

La edad media de los pacientes estudiados fue de $60 \pm 7,8$ años. La Figura 1 muestra el porcentaje de casos detectados por las diferentes técnicas diagnósticas.

Según los resultados del gated-SPECT se evidenció isquemia miocárdica silente en 27 pacientes 


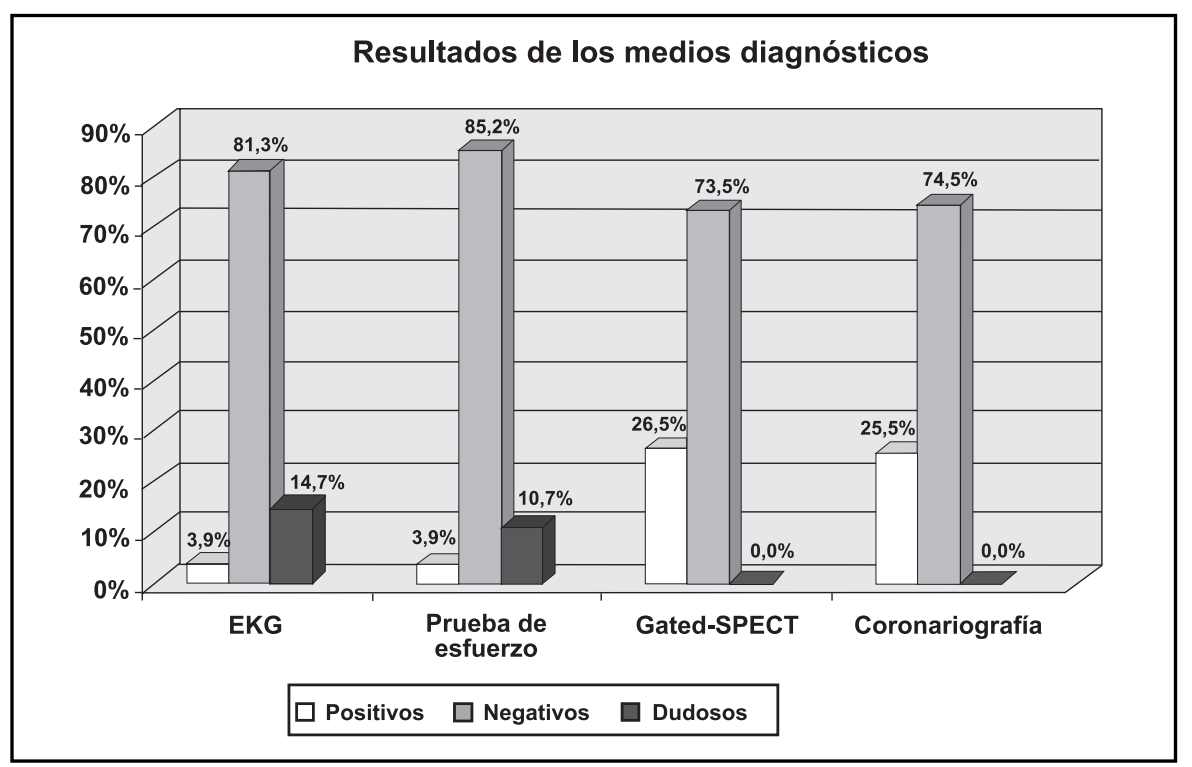

Figura 1. Porcentaje de isquemia miocárdica silente en la muestra estudiada, según las diferentes técnicas empleadas en el estudio.

(26,5\% de la población estudiada), en los 75 restantes $(73,5 \%)$ los resultados de los estudios de perfusión fueron normales. El 43,2\% mostró defecto de captación en más de un territorio, 48,2\% fueron defectos reversibles de intensidad ligera, 44,4\% defectos reversibles de intensidad moderada y severa. En dos de los casos $(7,4 \%)$ se reportó defecto severo no reversible (necrosis). El análisis funcional reveló que la media de los valores de la fracción de eyección (FE) fue significativamente inferior en el grupo con isquemia silente en relación con el grupo sin isquemia ( $47 \%$ versus $58,4 \%$ ), $\mathrm{p}=0,000$.

La coronariografía mostró obstrucción de las arterias coronarias en 26 pacientes (25,5\% del total de la población estudiada). En los 76 pacientes restantes los resultados fueron normales (74,5\%). El 57,6\% de las coronariografias con obstrucciones $\geq 50 \%$ reportaron enfermedad de un vaso. El 42,4\% notificó enfermedad multivaso. La Tabla 1 muestra la clasificación cruzada entre los resultados de la coronariografía y el gated-SPECT. El gated-SPECT reportó 24 verdaderos positivos, 3 falsos positivos, 2 falsos negativos y 73 verdaderos negativos. Los valores de sensibilidad, especificidad, exactitud, valor predictivo positivo y valor predictivo negativo fueron de 92,3\%, 96\%, 95\%, 88,8\%, 97,3\%, respectivamente. Existió buena correspondencia entre los resultados angiográficos y los del
gated-SPECT con un coeficiente de contingencia de 0,658, una $\mathrm{p}=0,000$ y un coeficiente Kappa de 0,873. Las Figuras 2a y 2b muestran imágenes de gatedSPECT y coronariografía de un paciente diabético tipo 2 con isquemia miocárdica silente.

El electrocardiograma basal evidenció cuatro pacientes con isquemia miocárdica silente (3,9\%), 15 resultados dudosos o con alteraciones inespecíficas $(14,7 \%)$ y 83 resultados normales $(81,3 \%)$. La Tabla 2 muestra la comparación cruzada entre electrocardiograma basal y gated-SPECT. En el grupo con gated-SPECT positivo existió 14,8\% de electrocardiogramas basales positivos. El 80\% de los electrocardiogramas basales con resultados dudosos de cardiopatía isquémica tuvieron un gatedSPECT con isquemia miocárdica silente. El 86,7\% de los electrocardiogramas negativos mostraron gated-SPECT negativo de isquemia. El coeficiente de contingencia fue de 0,534 con $\mathrm{p}=0,000$.

La prueba de esfuerzo mostró seis casos con isquemia miocárdica silente $(5,9 \%), 10$ con resultados dudosos o no concluyentes $(9,8 \%)$ y $86(84,3 \%)$ resultados negativos de isquemia. La Tabla 3 muestra la comparación cruzada entre la prueba de esfuerzo y el gated-SPECT. En el grupo con gated-SPECT positivo existió $18,5 \%$ de pruebas de esfuerzo positivas. El 70\% de las pruebas de esfuerzo dudosas 


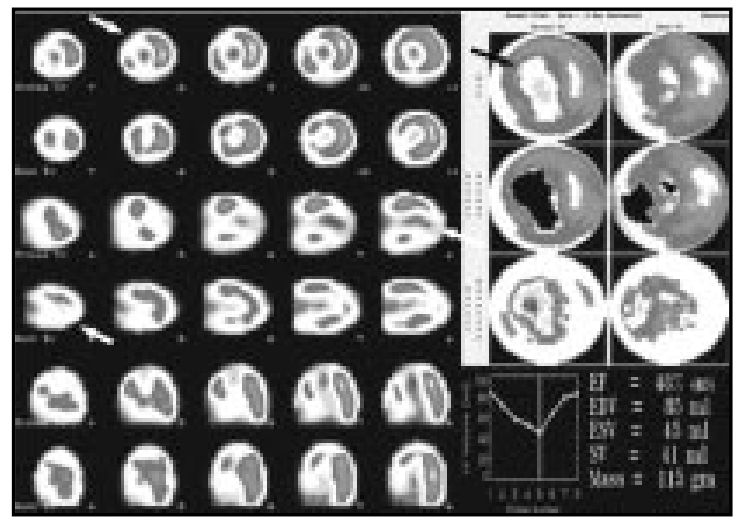

$2 \mathrm{a}$

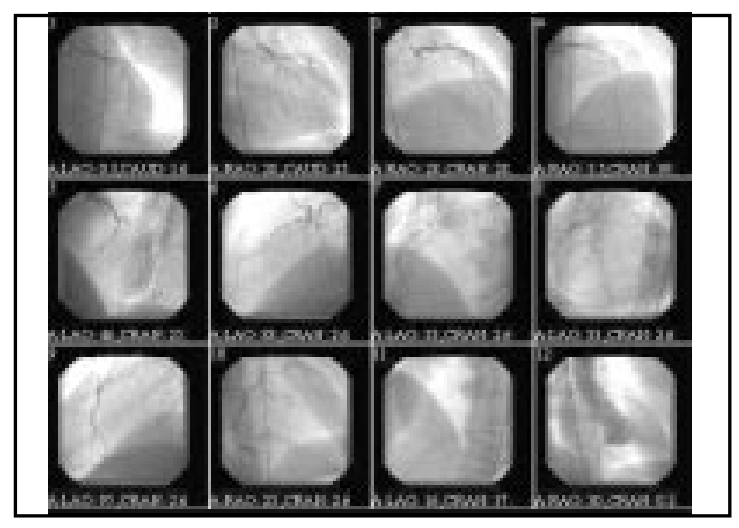

$2 \mathrm{~b}$

Figura 2. Paciente masculino de 57 años, mestizo, con 7 años de evolución de la diabetes e hipertenso. a) Sección de la izquierda: Cortes de Gated-SPECT: Eje corto, eje largo vertical y eje largo horizontal. Se observan lesiones isquémicas en las regiones anterior-septal-apical-moderada e inferior-septal-apical-severa (enfermedad multivasos). Sección de la derecha superior: Mapas polares en 2 columnas. Columna izquierda estudio de estrés y columna de la derecha estudio en reposo. Fila superior: mapas de los estudios de perfusión. Fila intermedia: mapas de la extensión de los defectos. Fila inferior: mapas de la severidad de los defectos. Sección derecha inferior: Resultados del análisis funcional. b) Cortes angiográficos del mismo paciente muestran estenosis mayores de 50\% del árbol coronario en las arterias descendente anterior y coronaria derecha.

Tabla 1. Clasificación cruzada entre los resultados del gated-SPECT y la coronariografía

\begin{tabular}{|lcccc|}
\hline Gated-SPECT & \multicolumn{2}{c|}{ Coronariografía } & Total \\
\hline \multirow{2}{*}{ Positivo } & Positiva & Negativa & 27 \\
& $\mathrm{~N}$ & 24 & 3 & $100,0 \%$ \\
Negativo & $\%$ & $88,9 \%$ & $11,1 \%$ & 75 \\
& $\mathrm{~N}$ & 2 & 73 & $100,0 \%$ \\
Total & $\%$ & $2,7 \%$ & $97,3 \%$ & 102 \\
& $\mathrm{~N}$ & 26 & 76 & $100,0 \%$ \\
\hline
\end{tabular}

Coeficiente de contingencia $=0,658 ; \mathrm{p}=0,000 ;$ Kappa $=0,873$.

tuvieron isquemia miocárdica silente según resultados del gated-SPECT. El 82,6\% de las pruebas de esfuerzo negativas mostraron un gated-SPECT negativo de isquemia. El coeficiente de contingencia fue de 0,404 con $\mathrm{p}=0,001$.

\section{DisCUSIÓN}

Según American College of Cardiology y American Society of Nuclear Cardiology, se recomienda el
gated-SPECT de esfuerzo como prueba inicial en pacientes de alto riesgo (diabéticos o con riesgo de complicaciones $>20 \%$ en 10 años) a modo de clase IIa y nivel de evidencia B?

Estudios anteriores reportan una prevalencia de isquemia miocárdica silente en el diabético entre $9 \%$ y $57 \% \%^{3-5,14-17}$. En la muestra estudiada se encontró $26,5 \%$ de isquemia miocárdica silente por resultados del gated-SPECT y 25,5\% según coronariografía. Esto corrobora que la isquemia miocárdica silente es frecuente en el diabético 
Tabla 2. Comparación cruzada entre los resultados del gated-SPECT y el electrocardiograma basal

\begin{tabular}{|c|c|c|c|c|c|}
\hline & & \multicolumn{4}{|c|}{ G ated-SPECT } \\
\hline & & & Positivo & Negativo & Total \\
\hline \multirow[t]{9}{*}{ EKG } & Positivo & $\mathrm{N}$ & 4 & 0 & 4 \\
\hline & & \% con $\mathrm{EKG}$ & $100,0 \%$ & $0,0 \%$ & $100,0 \%$ \\
\hline & & $\%$ con SPECT & $14,8 \%$ & $0,0 \%$ & $3,9 \%$ \\
\hline & Dudoso* & $\mathrm{N}$ & 12 & 3 & 15 \\
\hline & & $\%$ con $\mathrm{EKG}$ & $80,0 \%$ & $20,0 \%$ & $100,0 \%$ \\
\hline & & $\%$ con SPECT & $44,4 \%$ & $4,0 \%$ & $14,7 \%$ \\
\hline & Negativo & $\mathrm{N}$ & 11 & 72 & 83 \\
\hline & & \% con EKG & $13,3 \%$ & $86,7 \%$ & $100,0 \%$ \\
\hline & & $\%$ con SPECT & $40,7 \%$ & $96,0 \%$ & $81,4 \%$ \\
\hline \multirow[t]{3}{*}{ Total } & & $\mathrm{N}$ & 27 & 75 & 102 \\
\hline & & $\%$ con $\mathrm{EKG}$ & $26,5 \%$ & $73,5 \%$ & $100,0 \%$ \\
\hline & & $\%$ con SPECT & $100,0 \%$ & $100,0 \%$ & $100,0 \%$ \\
\hline
\end{tabular}

*Con alteraciones inespecíficas de cardiopatía isquémica. Coeficiente de contingencia =0,534; p =0,000.

Tabla 3. Clasificación cruzada entre los resultados del gated-SPECT y la Prueba de esfuerzo

\begin{tabular}{|c|c|c|c|c|}
\hline \multirow[b]{2}{*}{ Prueba de esfuerzo } & \multicolumn{4}{|c|}{ G ated-SPECT } \\
\hline & & Positivo & Negativo & Total \\
\hline \multirow[t]{3}{*}{ Positiva } & $\mathrm{N}$ & 5 & 1 & 6 \\
\hline & \% P. esfuerzo & $83,3 \%$ & $16,7 \%$ & $100,0 \%$ \\
\hline & $\%$ SPECT & $18,5 \%$ & $1,3 \%$ & $5,9 \%$ \\
\hline \multirow[t]{3}{*}{ Dudosa } & $\mathrm{N}$ & 7 & 3 & 10 \\
\hline & \% P. esfuerzo & $70,0 \%$ & $30,0 \%$ & $100,0 \%$ \\
\hline & $\%$ SPECT & $25,9 \%$ & $4,0 \%$ & $9,8 \%$ \\
\hline \multirow[t]{3}{*}{ Negativa } & $\mathrm{N}$ & 15 & 71 & 86 \\
\hline & \% P. esfuerzo & $17,4 \%$ & $82,6 \%$ & $100,0 \%$ \\
\hline & $\%$ SPECT & $55,6 \%$ & $94,7 \%$ & $84,3 \%$ \\
\hline \multirow[t]{3}{*}{ Total } & $\mathrm{N}$ & 27 & 75 & 102 \\
\hline & \% P. esfuerzo & $26,5 \%$ & $73,5 \%$ & $100,0 \%$ \\
\hline & $\%$ SPECT & $100,0 \%$ & $100,0 \%$ & $100,0 \%$ \\
\hline
\end{tabular}

Coeficiente de contingencia $=0,404 ; \mathrm{p}=0,000$.

tipo 2, aun cuando no existen signos clínicos evidentes de la enfermedad.

El gated-SPECT constituye una herramienta eficaz para predecir la enfermedad estenótica arterial coronaria y la severidad de las estenosis en el paciente con clínica cardiovascular ${ }^{18-20}$, no obstante, debido a su costo no es utilizado como método de screening en pacientes asintomáticos de alto riesgo. Investigaciones anteriores han validado la determinación de la fracción de eyección del ventrículo izquierdo y los volúmenes cardiacos empleando el gated-SPECT ${ }^{18,19}$. Este método permite la identificación mejorada de artefactos, de tejido blando y refuerza el descubri- 
miento de la enfermedad multivaso de las arterias coronarias. Además esta técnica proporciona una información poderosa para la valoración del riesgo de los pacientes con enfermedad arterial coronaria conocida o sospechosa y ayuda en la valoración de la viabilidad del miocardio ${ }^{15,17}$.

En el presente estudio se confirmó la alta capacidad diagnóstica del gated-SPECT, se obtuvieron adecuados valores de sensibilidad, especificidad, exactitud, VPP y VPN y una buena correspondencia con los estudios angiográficos $(\mathrm{k}$ $=0,873$ ). Se hallaron tres pacientes con resultados falsos positivos, cuyo denominador común fue la hipertensión arterial de más de 10 años de evolución.

Es conocido que existen situaciones fisiológicas que son causas de hipoactividad regional y que deben tenerse en cuenta en la interpretación de las imágenes porque pueden ser causa de falsos positivos, las más frecuentes son la hipertrofia ventricular causada por hipertensión arterial, la atenuación mamaria, la atenuación diafragmática y los bloqueos de rama izquierda.

Es ineludible recordar que la perfusión miocárdica es una evaluación funcional que comprende el árbol coronario completo mientras que la angiografía es un estudio anatómico cuya mejor información es sobre las arterias epicárdicas. Existen causas de isquemia sin lesión epicárdica, entre ellas: consumo de cocaína, lesiones de la microcirculación en pacientes diabéticos o con enfermedades del colágeno y puentes musculares. Estas situaciones clínicas ocasionan perfusiones positivas sin lesiones epicárdicas que obligan a contar el resultado de la perfusión miocárdica para el cálculo de las características operativas como "falso positivo" en un sujeto que en realidad sí tiene riesgo coronario secundario a la enfermedad de base.

En la muestra estudiada hallamos dos pacientes con resultados falsos negativos, se valoró la enfermedad multivaso como posible causa. La obstrucción de varias arterias provoca una enfermedad coronaria difusa con isquemia homogénea en todo el ventrículo izquierdo en la que no se puede distinguir alguna región más hipocaptante que el resto en las imágenes de perfusión, esto se presta para falsos resultados ${ }^{21,22}$. Un número importante de los pacientes mostraron obstruc- ción de dos arterias y ambos casos falsos negativos fueron reportados con posterioridad por coronariografía como enfermedad multivaso. Candell-Riera y cols ${ }^{23}$ estudiaron recientemente la significación de la SPECT negativa en pacientes con prueba de esfuerzo positiva, ellos encontraron que esta asociación es infrecuente y que la mayoría de los estudios falsos negativos eran debido a enfermedad multivaso o del tronco común de las arterias coronarias.

Un número substancial de los pacientes con electrocardiograma y prueba de esfuerzo de resultados dudosos mostraron un gated-SPECT con isquemia miocárdica silente. El electrocardiograma basal evidenció mayor asociación con los resultados del gated-SPECT que la prueba de esfuerzo. Estos resultados concuerdan con algunas investigaciones realizadas que han manifestado que existe correspondencia entre el electrocardiograma y el SPECT ${ }^{19}$. Otros autores han reportado un valor predictivo positivo bajo para la SPECT en pacientes con electrocardiograma negativo aun cuando éstos tenían dolor torácico ${ }^{20}$. De Santiago y $\operatorname{cols}^{24}$ estudiaron el valor pronóstico del electrocardiograma en pacientes diabéticos sin enfermedad cardiovascular conocida y concluyeron que las alteraciones en el electrocardiograma pueden predecir la aparición de eventos cardiovasculares con mayor precisión que los factores de riesgos solos. Esto podría ser de interés para seleccionar subpoblaciones de diabéticos de mayor riesgo.

La diabetes mellitus tipo 2 es considerada en nuestros días como sinónimo de enfermedad cardiovascular, es necesario, desde nuestro punto de vista, insistir en el cuidado que se debe tener con los pacientes diabéticos tipo 2 ante resultados dudosos en los estudios electrocardiográficos por la alta probabilidad de isquemia silente que éstos pueden tener según los actuales resultados. Estudios con muestras mayores serían necesarios para obtener mejores conclusiones al respecto.

Conclusiones: El gated-SPECT fue útil para la detección de isquemia miocárdica silente. Los resultados dudosos en el electrocardiograma basal y la prueba de esfuerzo sugieren en el diabético tipo 2 la necesidad de indicar estudios más sensibles y específicos. 


\section{REFERENCIAS}

1. Tabibiazar R, Edelman SV. Silent ischemia in people with diabetes: A condition that must be heard. Clinical Diabetes 2003; 21: 5-9.

2. Evans JMM, Barnett KN, McMurdo MET, Morris AD. Reporting of diabetes on death certificates of 1872 people with type 2 diabetes in Tayside, Scotland. Eur J Public Health 2008; 18: 201-3.

3. Wackers FJ, Young LH, Inzucchi SE, Chyun DA, Davey JA, Barrett EJ ET al. Detection of Silent Myocardial Ischemia in Asymptomatic Diabetic Subjects. The DIAD study. Diabetes Care 2004; 27: 1954-61.

4. Sajadien A, Nielsen OW, Rasmussen V, Hein HO, Hansen JF. Prevalence and prognostic significance of dailylife silent myocardial ischemia in middle-aged and elderly subjects with no apparent heart disease. Eur Heart J 2005; 26: 1402-9.

5. Val Gómez M, Gallardo FG, García A, San Martín Ma, TERol I. Isquemia silente versus angina en la tomogammagrafía con Tl-201. Rev Esp Med Nucl 2004; 23: 267-72.

6. Nieto R, Martín A, Pereztol O. Estudios Gated-SPeCT en Cardiología Nuclear. Aspectos técnicos. In: José M. Castro-Beiras (Ed) Cardiología Nuclear y otras Técnicas no invasivas de imagen en Cardiología. Medi Técnica, S.L. Madrid, España 2005; 115-121.

7. Schepis T, Gaemperli O, Koepfli P, Valenta I, Strobel K, Brunner A ET al. Comparison of 64-Slice CT with Gated SPECT for Evaluation of Left Ventricular Function. J Nucl Med 2006; 47: 1288-94.

8. Emmett L, Iwanochko RM, Freeman Mr, Barolet A, Lee DS, Husain M. Reversible regional wall motion abnormalities on exercise technetium-99m-gated cardiac single photon emission computed tomography predict high-grade angiographic stenoses. J Am College of Cardiology 2002; 39: 991-8.

9. Candell-Riera J, De león G, Jurado-López JA, DiegoDomínguez M, Albert-Bertrand FX, Coma-Canellae I. Evidencias clínicas y recomendaciones de la gatedSPECT de perfusión miocárdica. Rev Esp Cardiol 2008; 8: 58B-64B.

10. Gibbons RJ, Balady GJ, Bricker JT, Chaitman BR, Fletcher GF, Froelicher VF et al. ACC/AHA 2002 guideline update for exercise testing: summary article: a report of the American College of Cardiology/American Heart Association Task Force on Practice Guidelines. Circulation 2002; 106: 1883-92.

11. American Diabetes Association: Standards of medical care for patients with diabetes mellitus (Position Statement). Diabetes Care 2003; 26 Supl 1: S33-S50.

12. Committee on Advanced Cardiac Imaging and Technology, Council on Clinical Cardiology, American Heart Association; Cardiovascular Imaging Committee, American College of Cardiology; and Board of Directors, Cardiovascular Council, Society of Nu- clear Medicine. Standardization of cardiac tomographic imaging. Circulation 1992; 86: 338-9.

13. Faber Tl, Cooke CD, Folks RD, Vansant JP, Nichols KJ, DePuey EG ET AL. Left ventricular function and perfusion from gated SPECT perfusion images: an integrated method. J Nucl Med 1999; 40: 650-9.

14. Xanthos T, Ekmektzoglou KA, Papadimitriou L. Reviewing myocardial silent ischemia: specific patient subgroups. Int J Cardiol 2008; 124: 139-48.

15. De lorenzo A, Lima Ronaldo SL, Siqueira-Filho AG, Pantoja MR. Prevalence and Prognostic Value of Perfusion Defects Detected by Stress Technetium99m Sestamibi Myocardial Perfusion Single-Photon Emission Computed Tomography in Asymptomatic Patients With Diabetes Mellitus and No Known Coronary Artery Disease. Am J Cardiol 2002; 90 : 827-32.

16. Barthelemy O, Le Feuvre C, Timsit J. Silent myocardial ischemia screening in patients with diabetes mellitus. Arq Bras Endocrinol Metabol 2007; 51: 285-93.

17. Peña Y, Fernández-Britto J, Bacallao J, Batista JF, Coca MA, Torrac N et al. Diagnóstico de isquemia miocárdica silente en diabéticos tipo 2 mediante electrocardiograma, ergometría y gated-SPECT. Rev Cub Invest Bioméd 2008; 27: 20-5.

18. Go V, Bhatt MR, Hendel RC. The diagnostic and prognostic value of ECG-gated SPECT myocardial perfusion imaging. J Nucl Med 2004; 45: 912-21.

19. Henzlova MJ, Croft LB. The electrocardiogram as a predictor of left ventricular systolic function: correlation with gated SPECT imaging. Mt Sinai J Med 2003; 70: 306-9.

20. Candell-Riera J, Oller-Martínez G, Pereztol-Valdés O, Castell-Conesa J, Aguadé-Bruix S, García Alonso C et AL. Early myocardial perfusion gated-SPECT in patients with chest pain and non-diagnostic ECG in the emergency department. Rev Esp Cardiol 2004; 57 : 225-33.

21. De León G, Aguadé-Bruix S. Recomendaciones sobre las maniobras de provocación de isquemia, protocolos de adquisición, interpretación de las imágenes y elaboración de los informes. Rev Esp Cardiol 2008; 8: 2B-14B.

22. Castell J, Santana C, Candell-Riera J, Aguadé S, Olona M, Canela T et al. La tomogammagrafía miocárdica de esfuerzo en el diagnóstico de la enfermedad coronaria multivaso. Rev Esp Cardiol 1997; 50: 635-42.

23. Candell-Riera J, Fernández C, Escudero F, De León G, Aguadé-Bruix S, Castell-Conesa J. Prevalencia y significado angiográfico de la SPECT de perfusión miocárdica normal con electrocardiograma de esfuerzo positivo. Rev Esp Cardiol 2004; 57: 894-7.

24. De Santiago A, García-Lledó A, Ramos E y Santiago C. Valor pronóstico de electrocardiograma en pacientes con diabetes tipo 2 sin enfermedad cardiovascular conocida. Rev Esp Cardiol 2007; 60: 1035-41. 